\title{
Top properties measurements with the ATLAS detector
}

\section{Tom Neep*}

On behalf of the ATLAS Collaboration

University of Manchester

E-mail: tom.neepdcern.ch

\begin{abstract}
The top quark is unique among the known quarks in that it decays before it has an opportunity to form hadronic bound states. This makes measurements of its properties particularly interesting as one can access directly the properties of a bare quark. Measurements of the charge asymmetry in $t \bar{t}$ pair events are presented. The measurements use the $7 \mathrm{TeV}$ ATLAS datasets and probe models of physics beyond the Standard Model. A measurement of the correlation between the direction of the spins of $t \bar{t}$ pairs is also presented. The measurement agrees with the Standard Model and is used to set limits on the production of the supersymmetric partner of the top quark. In addition, a novel measurement of colour flow in $t \bar{t}$ pair events is presented. The measurement uses the jets originating from the $W$ boson and demonstrates the ability of the colour flow observable to distinguish between colour octet and colour singlet final states.
\end{abstract}

The European Physical Society Conference on High Energy Physics

22-29 July 2015

Vienna, Austria

*Speaker. 


\section{Introduction}

The top quark has the largest mass of any fundamental particle in the Standard Model (SM), over a factor of 40 times greater than the second most massive quark, the bottom quark. An important consequence of this is the top quark decays before it can hadronise, allowing many of its properties to be studied. These properties include: the mass, charge and polarisation of the top quark, how it couples to other particles such as the Higgs boson and how the top quark decays, amongst others. The large number of top quarks produced at the LHC means that these properties can be tested to a higher precision than ever before, allowing to test SM predictions and to search for new physics Beyond the Standard Model (BSM).

Top quarks decay to a $b$-quark and $W$ boson $\approx 100 \%$ of the time, and thus top-antitop-quark pairs $(t \bar{t})$ are categorised based on how each of the two $W$ bosons decay. In this article, three measurements performed by the ATLAS collaboration [1] using top-quark pairs are discussed. Two of these measurements are performed in the dilepton channel, in which both $W$ bosons decay into a charged lepton and neutrino, while the third measurement uses the lepton-plus-jets channel, in which one of the $W$ bosons decays into a charged lepton and neutrino, while the other decays to two quarks.

\section{2. $t \bar{t}$ charge asymmetry}

$t \bar{t}$ production is predicted to be symmetric under charge conjugation at leading order in Quantum Chromodynamics (QCD) in the SM. However, at next-to-leading order a small $(\approx 1 \%)$ charge asymmetry is introduced resulting in the rapidity distriubtion of top quarks being slightly broader than the one for top anti-quarks. This asymmetry is passed on to the decay products of the top quark and thus also exists between the charged leptons in dilepton $t \bar{t}$ events. Although the $t \bar{t}$ charge asymmetry is predicted to be small in the SM, BSM physics can cause it to be enhanced.

The ATLAS collaboration has measured the $t \bar{t}$ charge asymmetry by reconstructing the $t \bar{t}$ system in dilepton $t \bar{t}$ events and also by directly using the charged leptons in such events [2]. The $t \bar{t}$ charge asymmetry is defined as

$$
A_{C}^{t \bar{t}}=\frac{N(\Delta|y|>0)-N(\Delta|y|<0)}{N(\Delta|y|>0)+N(\Delta|y|<0)}
$$

where $\Delta|y|=\left|y_{t}\right|-\left|y_{\bar{t}}\right|,{ }^{1}$ while the lepton based charged asymmetry is defined as

$$
A_{C}^{\ell \ell}=\frac{N(\Delta|\eta|>0)-N(\Delta|\eta|<0)}{N(\Delta|\eta|>0)+N(\Delta|\eta|<0)}
$$

with $\Delta|\eta|=\left|\eta_{\ell^{+}}\right|-\left|\eta_{\ell^{-}}\right|$. Figure 1 shows comparisons of data to the SM prediction for the (a) $t \bar{t}$ charge asymmetry and (b) lepton based charge asymmetry. In both cases good agreement is seen between the data and expectation.

\footnotetext{
${ }^{1}$ ATLAS uses a right-handed coordinate system with its origin at the nominal interaction point (IP) in the centre of the detector and the $z$-axis along the beam pipe. The $x$-axis points from the IP to the centre of the LHC ring, and the $y$-axis points upward. Cylindrical coordinates $(r, \phi)$ are used in the transverse plane, $\phi$ being the azimuthal angle around the beam pipe. The pseudorapidity is defined in terms of the polar angle $\theta$ as $\eta=-\ln \tan (\theta / 2)$.
} 


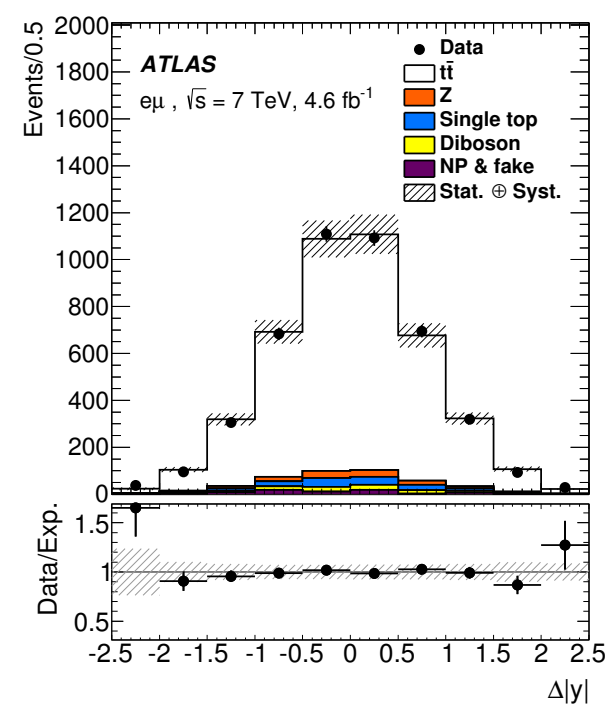

(a)

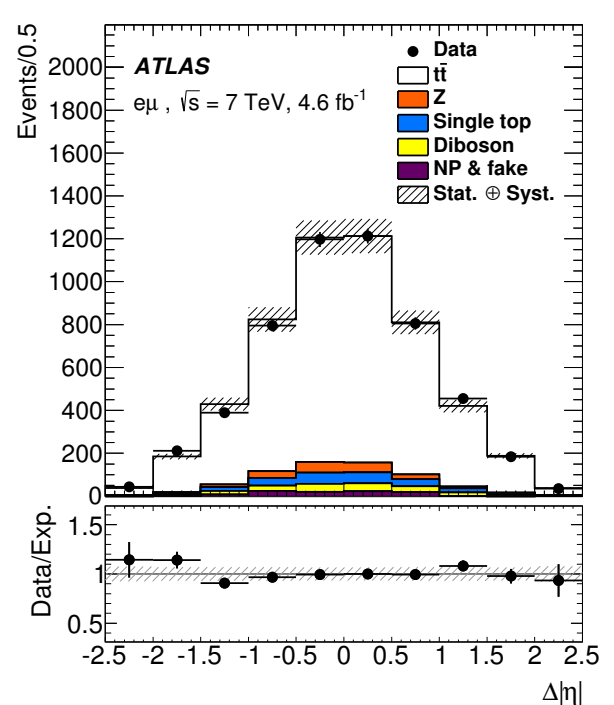

(b)

Figure 1: Comparisons of the SM prediction to data for the (a) $\Delta|y|$ and (b) $\Delta|\eta|$ distributions. Taken from [2].

Each of these distributions is unfolded to correct for detector reconstruction and acceptance effects. The unfolded distributions are shown in Figure 2. The number of bins is determined by the resolution of each variable. The reconstruction of the $t \bar{t}$ system required to obtain $\Delta|y|$ results in a lower resolution than the lepton based charge asymmetry and thus fewer bins can be used. The

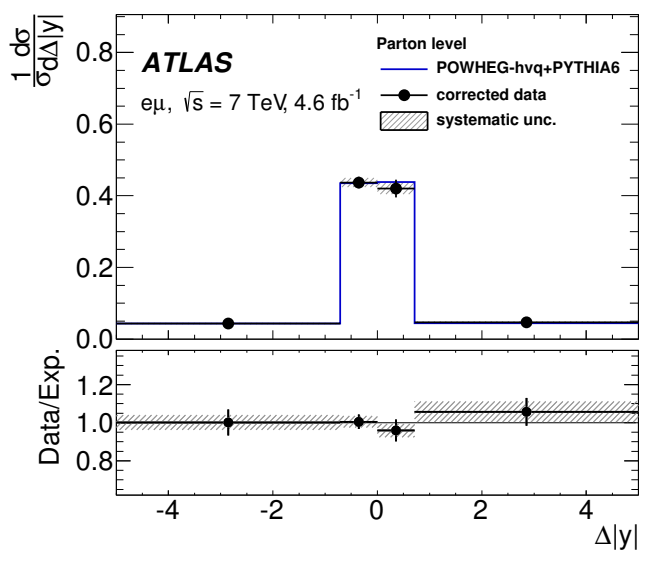

(a)

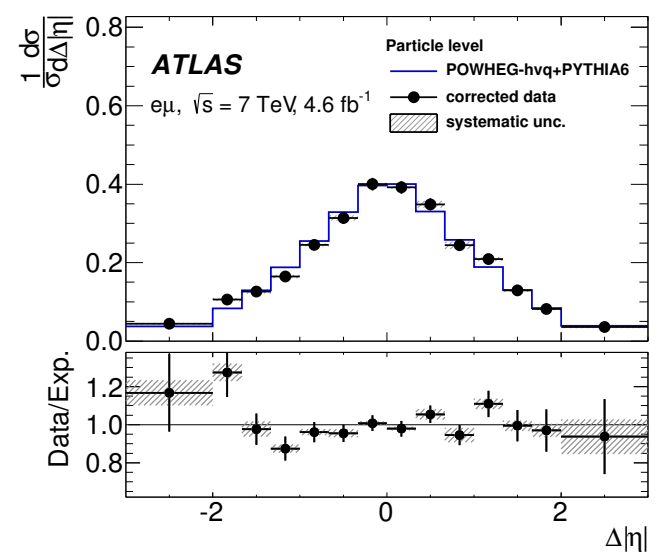

(b)

Figure 2: Comparisons of unfolded data to the SM prediction for the (a) $\Delta|y|$ and (b) $\Delta|\eta|$ distributions after correcting for detector and acceptance effects. Taken from [2].

inclusive $t \bar{t}$ and lepton-based charge asymmetries, $A_{C}^{t \bar{t}}$ and $A_{C}^{\ell \ell}$, are also unfolded. The results are shown graphically in Figure 3. The measured values of $A_{C}^{t \bar{t}}$ and $A_{C}^{\ell \ell}$ are in good agreement with the $\mathrm{SM}$ prediction at next-to-leading order in QCD including electroweak corrections [3]. 


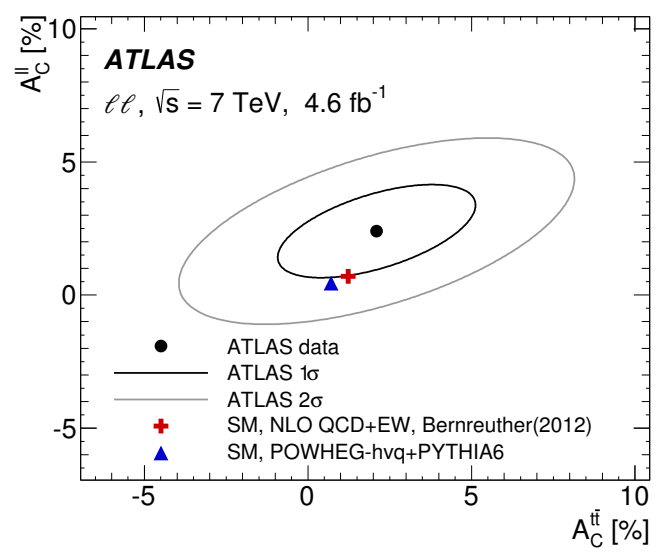

Figure 3: The measured value of $A_{C}^{t \bar{t}}$ and $A_{C}^{\ell \ell}$ compared to SM predictions. The results are in good agreement with the predictions. Taken from [2].

\section{3. $t \bar{t}$ spin correlation}

Top quarks produced in pairs via the strong interaction have essentially unpolarised spins. However the spins of the top quark and anti-top quark are expected to be correlated. Information about the spins of the top and anti-top quark is passed on to their decay products as the tops decay before they can hadronise and the strength of the spin correlation can be measured using angular distributions.

At the LHC, where $t \bar{t}$ pairs are primarily produced via gluon-gluon fusion, the correlation affects the azimuthal angle in the lab frame between the two charged leptons in dilepton $t \bar{t}$ events, $\Delta \phi\left(\ell^{+}, \ell^{-}\right)$[4]. Dilepton $t \bar{t}$ events are particularly sensitive to spin correlations as charged leptons carry approximately $100 \%$ of the information about the top quark spin and can be identified with a high efficiency by the ATLAS detector. Additionally, dilepton $t \bar{t}$ events can be selected with very low backgrounds by requiring jets to be $b$-tagged.

ATLAS has measured the $\Delta \phi\left(\ell^{+}, \ell^{-}\right)$distribution in dilepton $t \bar{t}$ events [5]. The expected $\Delta \phi\left(\ell^{+}, \ell^{-}\right)$distribution for $t \bar{t}$ events is shown in Figure 4(a) for the case of SM spin correlation $(A=\mathrm{SM})$ and for the case of zero spin correlation $(A=0)$. A clear difference can be seen between the two scenarios, showing the sensitivity of this variable to spin correlation effects. These two scenarios are used as templates in a fit to data to extract a value of $f_{\mathrm{SM}}$, which is a measure of the spin correlation strength. If the data agreed perfectly with the SM template then $f_{\mathrm{SM}}=1$, while if it agreed perfectly with the zero spin correlation template then $f_{\mathrm{SM}}=0$. A value of $f_{\mathrm{SM}}>1$ implies a larger correlation than that predicted by the SM.

The fit to data is shown in Figure 4(b) by the dashed-red line and is in good agreement with the SM prediction while strongly disfavouring the zero spin correlation scenario. The prediction for top-squark pair production, $\tilde{\tilde{t}} \bar{t}$ is also shown on top of the SM prediction, for a top-squark mass of $180 \mathrm{GeV}$. If the stops decay to top quarks and light neutralinos then the $\tilde{t} \overline{\tilde{t}}$ final state would look very similar to the uncorrelated $t \bar{t}$ prediction and thus this analysis extends previous limits on top squark masses [6]. 


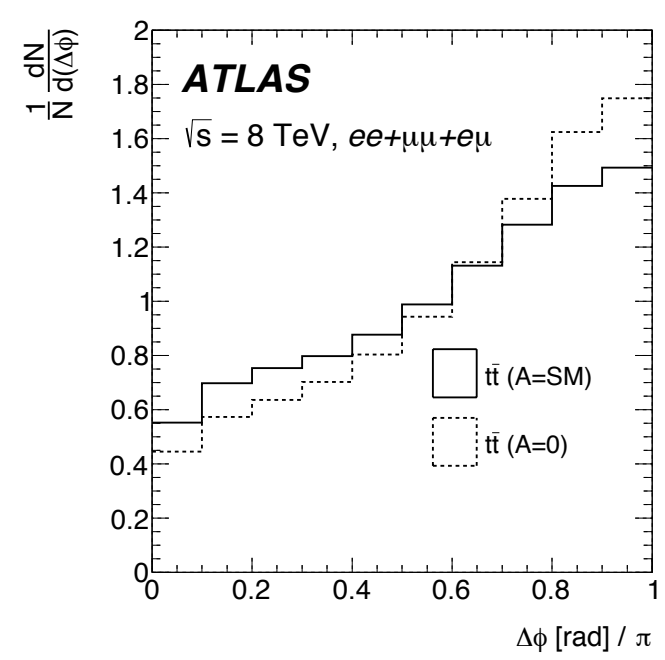

(a)

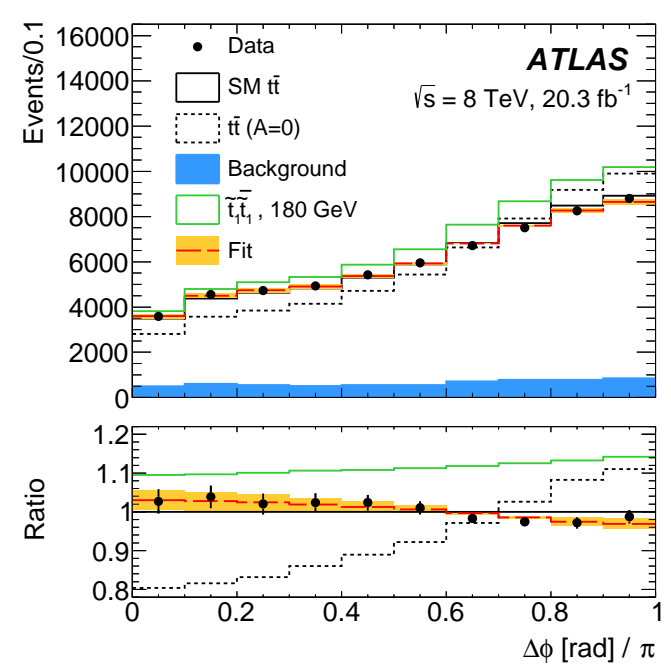

(b)

Figure 4: The (a) normalised $\Delta \phi\left(\ell^{+}, \ell^{-}\right)$distribution for SM $(A=\mathrm{SM})$ spin correlation and a scenario in which there is no spin correlation $(A=0)$, and (b) the comparison of data to the different predictions, including the scenario of top squark pairs. Taken from [5].

\section{Measurement of colour flow}

The distribution and orientation of jets provide information about colour connections between hard-scatter quarks and gluons. One variable predicted to be sensitive to the effects of colour connections is the jet pull angle [7]. ATLAS has measured the jet pull angle using hadronically decaying lepton-plus-jets $t \bar{t}$ events [8].

Events are selected that contain at least two $b$-tagged jets and at least four jets in total. The two highest- $p_{\mathrm{T}}$ non tagged jets are used to construct the jet pull angle. If these jets are colour connected, then the jet pull angle is predicted to peak at zero. The sensitivity to colour flow effects is demonstrated by comparing data to SM $t \bar{t}$ Monte Carlo (MC) and to modified $t \bar{t}$ MC in which the $W$ boson is exchanged for a colour octet. A schematic of the colour flow in these two scenarios is shown in Figure 5. Two pull angles are constructed using different jet constituents; inner detector
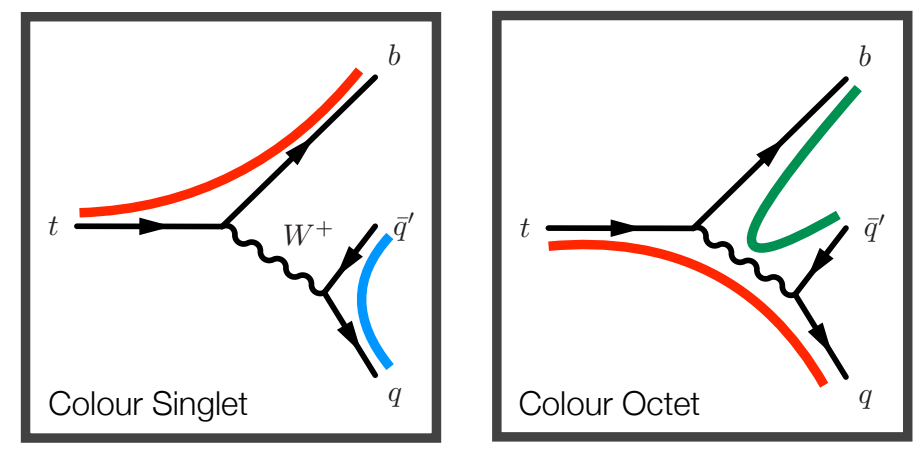

Figure 5: Schematic of the colour structures used in [8] to evaluate the sensitivity of the jet pull angle to colour flow. Taken from [8]. 
(ID) tracks and calorimeter clusters before these are both corrected for detector resolution and acceptance effects using an iterative Bayesian unfolding algorithm. The resulting distributions are shown in Figure 6 for (a) calorimeter clusters unfolded to all stable particles in the jet and (b) ID tracks unfolded to only charged particles in the jet. The data agree with the SM and disfavour

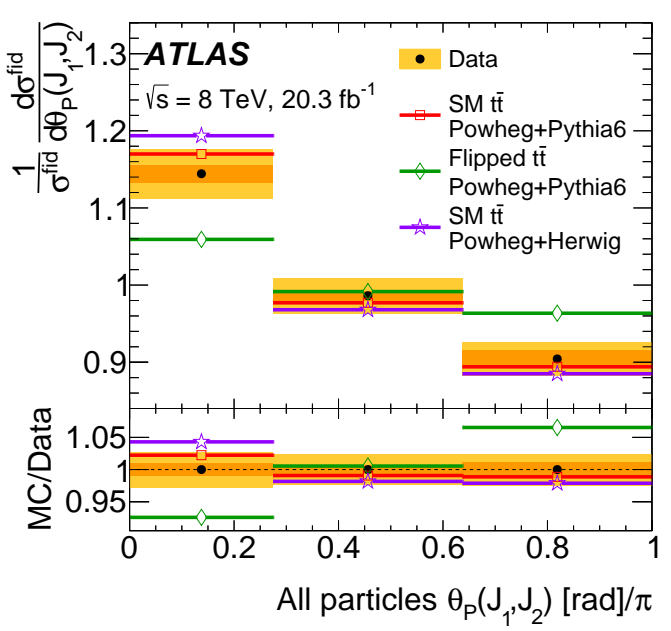

(a)

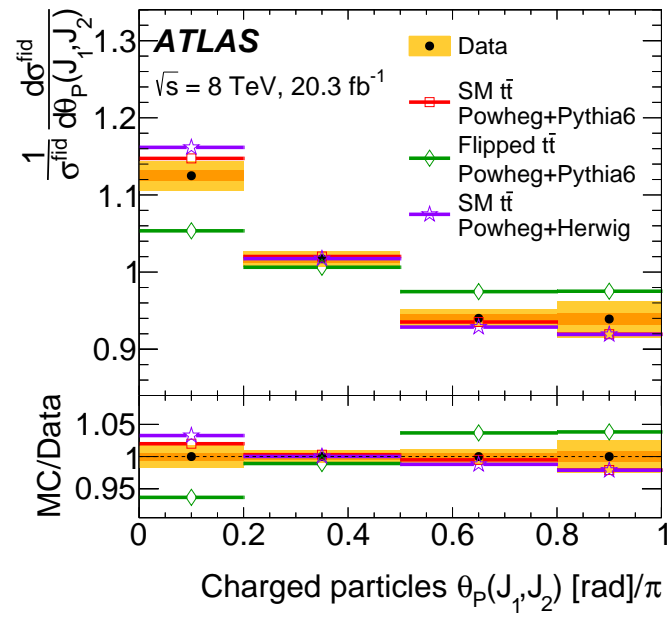

(b)

Figure 6: Comparison of data to $t \bar{t}$ predictions for the (a) all-particles and (b) charged-particles pull angle. Taken from [8].

the colour octet model at $>3 \sigma$ illustrating the potential to use the jet pull angle in future SM measurements and BSM searches.

\section{Conclusion and outlook}

Run 1 of the LHC has allowed the ATLAS detector to record a large data sample of $t \bar{t}$ events, allowing many properties of the top quark to be precisely measured.

Thus far, all measurements have been in agreement with the SM predictions and several models of BSM physics have been excluded using these measurements.

Run 2 of the LHC began this year and will deliver the largest sample of $t \bar{t}$ events ever, at the highest centre-of-mass energy ever, allowing the properties of the top quark to be measured to unprecedented accuracy.

\section{References}

[1] ATLAS Collaboration, JINST 3 (2008) S08003.

[2] ATLAS Collaboration, JHEP 05 (2015) 061, arXiv: 1501.07383 [hep-ex] .

[3] W. Bernreuther and Z. G. Si, Phys. Rev. D 86 (2012) 034026 [arXiv:1205.6580 [hep-ph]].

[4] G. Mahlon and S. J. Parke, Phys.Rev. D81 (2010) 074024, arXiv:1001.3422 [hep-ph] .

[5] ATLAS Collaboration, Phys. Rev. Lett. 114 (2015) 14, 142001, arXiv:1412.4742 [hep-ex]. 
[6] Z. Han, A. Katz, D. Krohn, and M. Reece, JHEP 1208 (2012) 083, arXiv: 1205.5808 [hep-ph].

[7] J. Gallicchio and M. D. Schwartz, Phys.Rev.Lett. 105 (2010) 022001, arXiv: 1001.5027 [hep-ph].

[8] ATLAS Collaboration, Physics Letters B (2015) 475-493 arXiv:1506.05629 [hep-ex] . 